\title{
On steady compressible flows with compact vorticity; the compressible Stuart vortex
}

\author{
By D. I. MEIRON ${ }^{1}$, D. W. MOORE AND D. I. PULLIN \\ ${ }^{1}$ California Institute of Technology, Pasadena, CA 91125, USA \\ ${ }^{2}$ Department of Mathematics, Imperial College of Science, Technology and Medicine, \\ Queens Gate London SW7 2BZ, UK
}

(Received 20 January 1999 and in revised form 1 June 1999)

Numerical and analytical solutions to the steady compressible Euler equations corresponding to a compressible analogue of the linear Stuart vortex array are presented. These correspond to a homentropic continuation, to finite Mach number, of the Stuart solution describing a linear vortex array in an incompressible fluid. The appropriate partial differential equations describing the flow correspond to the compressible homentropic Euler equations in two dimensions, with a prescribed vorticity-densitystreamfunction relationship. In order to construct a well-posed problem for this continuation, it was found, unexpectedly, to be necessary to introduce an eigenvalue into the vorticity-density-streamfunction equation. In the Rayleigh-Janzen expansion of solutions in even powers of the free-stream Mach number $M_{\infty}$, this eigenvalue is determined by a solvability condition. Accurate numerical solution by both finitedifference and spectral methods are presented for the compressible Stuart vortex, over a range of $M_{\infty}$, and of a parameter corresponding to a confined mass-flow rate. These also confirm the nonlinear eigenvalue character of the governing equations. All solution branches followed numerically were found to terminate when the maximum local Mach number just exceeded unity. For one such branch we present evidence for the existence of a very small range of $M_{\infty}$ over which smooth transonic shock-free flow can occur.

\section{Introduction}

This is the third paper in a sequence in which we study the effect of compressibility on steady incompressible vortical solutions of Euler's equations in two dimensions. Previous papers (Moore \& Pullin 1987, 1998) were devoted to the compressible version of the hollow vortex pair and the compressible version of Hill's spherical vortex respectively. In the present work we investigate the effect of compressibility on the Stuart vortex array. Ardalan, Meiron \& Pullin (1995) have studied the related problem, namely the hollow vortex array.

The Stuart vortex is a model of the inviscid mixing layer between two parallel streams moving with different speeds. To describe it we adopt axes $O x y$ moving with mean velocity of the two streams and represent the velocity field $(u(x, y), v(x, y))$ by a streamfunction $\psi(x, y)$ such that

$$
u=\frac{\partial \psi}{\partial y}, \quad v=-\frac{\partial \psi}{\partial x},
$$

with vorticity $\omega(x, y)$ given by

$$
\omega=-\nabla^{2} \psi
$$


Any steady vortical solution of the incompressible Euler equations has a constant vorticity along pathlines, so that

$$
\omega=F(\psi)
$$

The Stuart vortex corresponds to the choice (Stuart 1967)

$$
\omega=-\mathrm{e}^{-2 \psi},
$$

so that, in view of (1.2)

$$
\nabla^{2} \psi=\mathrm{e}^{-2 \psi} .
$$

The most general solution of (1.5) was found by Liouville: see Ames (1965), Forsyth (1959). Stuart (1967) found a family of exact solutions of (1.5) which are given by

$$
\psi_{0}=\ln \left(K \cosh y+\sqrt{K^{2}-1} \cos x\right),
$$

where $0 \leqslant K<\infty$ is a parameter defining the family. We note the following properties of (1.6).

(i) As $y \rightarrow \pm \infty$,

$$
\psi_{0} \sim y \operatorname{sgn}(y)+\ln \left(\frac{K}{2}\right)+O\left(\mathrm{e}^{-|y|}\right),
$$

so that

$$
u_{0} \sim \operatorname{sgn}(y), \quad v_{0} \sim 0
$$

which shows that the velocity asymptotes to opposed uniform streams each with unit speed when $y \rightarrow \pm 1$. The displacement thickness is $d=\ln (K / 2)$.

(ii) For $K>1 \psi_{0}$ is $2 \pi$-periodic with respect to $x$ and is even about the lines $x=0, \pm \pi, \pm 2 \pi \ldots$.

(iii) For $K>1$ the points with coordinates $(x=(2 n+1) \pi, y=0)$ are hyperbolic stagnation points and $(x=2 n \pi, y=0)$ are elliptical stagnation points, $n$ integer. The streamline pattern is thus a periodic array of cat's eyes, the dividing streamline being $\psi_{0}=\ln \left(K+\sqrt{K^{2}-1}\right)$.

The vorticity is $\omega=\left(K \cosh y+\sqrt{K^{2}-1} \cos x\right)^{-2}$ and it follows that the ratio of the vorticity at the elliptical stagnation points to that at the hyperbolic points is

$$
\left|\frac{\omega_{0}(\pi, 0)}{\omega_{0}(0,0)}\right|=\left(K+\sqrt{K^{2}-1}\right)^{4} .
$$

For $K=1$ this is unity corresponding to a solution consisting of a parallel shear flow

$$
u=\tanh y, \quad v=0 .
$$

(iv) When $K \rightarrow \infty$, the vorticity ratio is infinite and $\psi_{0}$ can be shown to describe the potential flow produced by an array of point vortices at $(x, y)=((2 n+1) \pi, 0)$, $n=-\infty, \ldots \infty$.

\section{The compressible Stuart vortex}

The governing equations are the compressible Euler equations

$$
\begin{gathered}
\boldsymbol{\omega} \times \boldsymbol{u}=-\nabla\left(\frac{1}{2} \boldsymbol{u}^{2}\right)-\frac{1}{\rho} \nabla p, \\
\nabla \cdot(\rho \boldsymbol{u})=0, \\
(\boldsymbol{u} \cdot \nabla) S=0 .
\end{gathered}
$$


Here $\boldsymbol{u}$ is the velocity, $\boldsymbol{\omega}$ is the vorticity, $S$ is the entropy, $p$ is the pressure and $\rho$ is the density.

We choose to satisfy (2.3) by insisting that $S=S_{0}$ where $S_{0}$ is independent of position. This will be seen to give a homentropic continuation of the Stuart vortex solution to finite Mach number; see Moore \& Pullin (1998) for a discussion of both this, and alternative continuation branches for the Hill's spherical vortex. For homentropic flow of a calorically perfect gas we have

$$
p=A \rho^{\gamma},
$$

where $A$ is independent of position and $\gamma$ is the ratio of specific heats. Equation (2.4) enables (2.1) to be written in the form

$$
\omega \times \boldsymbol{u}=-\nabla H,
$$

where

$$
H=\frac{1}{2} \boldsymbol{u}^{2}+\frac{\gamma}{\gamma-1} \frac{p}{\rho},
$$

is the total enthalpy. It follows from (2.5) that

$$
\boldsymbol{u} \cdot \nabla H=0,
$$

For plane motion, we can introduce a mass streamfunction $\psi(x, y)$ such that

$$
\rho u=\frac{\partial \psi}{\partial y}, \quad \rho v=-\frac{\partial \psi}{\partial x} .
$$

In view of (2.7), we have $H=H(\psi)$. Expressing the vorticity as $\boldsymbol{\omega}=\omega(x, y) \boldsymbol{k}$, where $\boldsymbol{k}$ is normal to the plane of flow, and taking components of (2.5) leads to

$$
\begin{aligned}
& \frac{\omega}{\rho} \frac{\partial \psi}{\partial x}=-\frac{\partial H}{\partial x}=-\frac{\partial \psi}{\partial x} \frac{\mathrm{d} H}{\mathrm{~d} \psi}, \\
& \frac{\omega}{\rho} \frac{\partial \psi}{\partial y}=-\frac{\partial H}{\partial y}=-\frac{\partial \psi}{\partial y} \frac{\mathrm{d} H}{\mathrm{~d} \psi} .
\end{aligned}
$$

so that

$$
\frac{\omega}{\rho}=-\frac{\mathrm{d} H}{\mathrm{~d} \psi} \equiv V(\psi),
$$

a special case of a relation obtained by Vazsonyi (1945).

We can now obtain equations for $\psi$ and $\rho$ by substitution of (2.8) into (2.11) and (2.6), with the result

$$
\begin{aligned}
\nabla^{2} \psi-\frac{1}{\rho}(\nabla \psi \cdot \nabla \rho) & =-\rho^{2} V(\psi), \\
\frac{1}{2 \rho^{2}}(\nabla \psi)^{2}+\frac{A \gamma \rho^{\gamma-1}}{\gamma-1} & =H_{0}-\int_{\infty}^{\psi} V\left(\psi^{\prime}\right) \mathrm{d} \psi^{\prime},
\end{aligned}
$$

where $H_{0}$ is a constant of integration.

We have non-dimensionalized the equations to conform with the scheme implicit in our description of the Stuart vortex, with the additional proviso that $\rho \rightarrow 1$ as $|y| \rightarrow \infty$. Then $\gamma A=M_{\infty}^{-2}$, where $M_{\infty}$ is the Mach number of the flow at infinity. If we let $|y| \rightarrow \infty$ we find that (2.13) implies

$$
\frac{1}{2}+\frac{1}{M_{\infty}^{2}(\gamma-1)}=H_{0},
$$


so that (2.13) then becomes

$$
\frac{M_{\infty}^{2}}{2}(\nabla \psi)^{2}+\frac{\rho^{2}\left(\rho^{\gamma-1}-1\right)}{\gamma-1}=\frac{M_{\infty}^{2} \rho^{2}}{2}\left(1-2 \int_{\infty}^{\psi} V\left(\psi^{\prime}\right) \mathrm{d} \psi^{\prime}\right) .
$$

At first glance, (2.12) together with (2.15) may appear to be of the elliptic type, owing to the presence of the $\nabla^{2}$ operator in (2.12). Since, however, these equations are a form of the steady inviscid rotational Euler equations with a prescribed vorticity-density relation, they must of course be of the mixed elliptic-hyperbolic type. This can be verified by differentiating (2.15) with respect to $x$ and solving for $\partial \rho / \partial x$. Repeating this by differentiating with respect to $y$ and subsequent substitution of the expressions for $\partial \rho / \partial x$ and $\partial \rho / \partial y$ into (2.12) then gives, after some algebra, a form of the vorticitystreamfunction equation which can be shown to be of the elliptic-hyperbolic type by standard methods.

We must now consider the choice of the function $V(\psi)$. We first tried the obvious choice $V(\psi)=-\exp (-2 \psi)$, but, as we discuss later, this choice proved over-restrictive. We were thus led to the choice

$$
V(\psi)=-\mathrm{e}^{-2 \mu \psi},
$$

where $\mu$ is to be found as part of the solution; of course $\mu \rightarrow 1$ as $M_{\infty} \rightarrow 0$. The vorticity parameter $\mu$ can be thought of as a nonlinear eigenvalue for the Stuart vortex. The vorticity and energy equations (2.12) and (2.15) now take their final form

$$
\begin{aligned}
\nabla^{2} \psi-\frac{1}{\rho}(\nabla \psi \cdot \nabla \rho) & =\rho^{2} \mathrm{e}^{-2 \mu \psi}, \\
\frac{M_{\infty}^{2}}{2}(\nabla \psi)^{2}+\frac{\rho^{2}\left(\rho^{\gamma-1}-1\right)}{\gamma-1} & =\frac{M_{\infty}^{2} \rho^{2}}{2}\left(1-\frac{1}{\mu} \mathrm{e}^{-2 \mu \psi}\right) .
\end{aligned}
$$

With computational efficiency in mind, we use the symmetries to reduce the domain in which we seek a solution to the semi-infinite rectangle $R$ defined by $0 \leqslant x \leqslant \pi$, $0 \leqslant y \leqslant \infty$. The boundary conditions are

$$
\begin{gathered}
\frac{\partial \psi}{\partial y}=0 \quad(y=0,0 \leqslant x \leqslant \pi), \\
\frac{\partial \psi}{\partial x}=0 \quad(x=0,0 \leqslant y \leqslant \infty) \quad \text { and } \quad(x=\pi, 0 \leqslant y \leqslant \infty), \\
\psi \sim y+d \quad \text { as } y \rightarrow \infty \quad(0 \leqslant x \leqslant \pi), \\
\rho \rightarrow 1 \quad \text { as } y \rightarrow \infty \quad(0 \leqslant x \leqslant \pi) .
\end{gathered}
$$

The displacement thickness $d\left(M_{\infty}^{2}\right)$ is unknown, as is $\mu\left(M_{\infty}^{2}\right)$ but we have $d(0)=$ $\ln (K / 2), \mu(0)=1$. We remark that there are no boundary conditions on $\rho$ on the sides and base of $R$. This is because $\partial \psi / \partial x=0$ implies $\partial \rho / \partial x=0$ and $\partial \psi / \partial y=0$ implies $\partial \rho / \partial y=0$; this follows from differentiating (2.18) with respect to $x$ and with respect to $y$.

The boundary conditions are incomplete because there is no condition which determines the degree of concentration of the vorticity. We chose to remedy this defect by specifying the mass flux within the cat's eyes; thus we put

$$
\psi(0,0)-\psi(\pi, 0)=f
$$


where $f$ is prescribed. For the incompressible Stuart vortex,

$$
\psi(0,0)-\psi(\pi, 0)=2 \ln \left(K+\sqrt{K^{2}-1}\right),
$$

so that we can define an effective $K$ for the Stuart vortex by

$$
f=2 \ln \left(K+\sqrt{K^{2}-1}\right) .
$$

When $K$ is referred to subsequently, (2.25) is implied. We remark that when $K=1$, $f=0$, the incompressible solution (1.6), (1.10) with $\rho=1$ is an exact solution of both (2.17) and (2.18) for all $M_{\infty}$.

\section{The Rayleigh-Janzen expansion}

In this section we seek an approximate solution to the governing equations (2.17)(2.18) by expanding the dependent variables in powers of the Mach number. Thus we introduce the expansions

$$
\begin{gathered}
\psi=\psi_{0}+M_{\infty}^{2} \psi_{1}+M_{\infty}^{4} \psi_{2}+\ldots, \\
\rho=1+M_{\infty}^{2} \rho_{1}+M_{\infty}^{4} \rho_{2}+\ldots, \\
\mu=1+M_{\infty}^{2} \mu_{1}+M_{\infty}^{4} \mu_{2}+\ldots
\end{gathered}
$$

The first term in each expansion corresponds to the Stuart vortex and in particular

$$
\psi_{0}=-\frac{1}{2} \ln \left(1-\epsilon^{2}\right)+\ln (\cosh y+\epsilon \cos x),
$$

where we have replaced $K$ by the parameter

$$
\epsilon=\frac{\sqrt{K^{2}-1}}{K}
$$

note that $K \rightarrow 1$ corresponds to $\epsilon \rightarrow 0$ and $K \rightarrow \infty$ corresponds to $\epsilon \rightarrow 1$.

Substitution of (3.1) and (3.2) into the energy equation (2.18) leads to

$$
\rho_{1}=\frac{1}{2}-\frac{1}{2} \mathrm{e}^{-2 \psi_{0}}-\frac{1}{2}\left(\nabla \psi_{0}\right)^{2} .
$$

Use of (3.4) in (3.6) then gives

$$
\rho_{1}=\frac{\epsilon \cos x}{(\cosh y+\epsilon \cos x)} .
$$

We can now obtain the equations determining $\psi_{1}$, which are

$$
\nabla^{2} \psi_{1}+2 \mathrm{e}^{-2 \psi_{0}} \psi_{1}=\nabla \rho_{1} \cdot \nabla \psi_{0}+2 \mathrm{e}^{-2 \psi_{0}}\left(\rho_{1}-\mu_{1} \psi_{0}\right),
$$

with boundary conditions

$$
\begin{gathered}
\frac{\partial \psi_{1}}{\partial y}=0 \quad \text { on }(y=0,0 \leqslant x \leqslant \pi), \\
\frac{\partial \psi_{1}}{\partial x}=0 \quad \text { on }(x=0,0 \leqslant y \leqslant \infty) \text { and }(x=\pi, 0 \leqslant y \leqslant \infty), \\
\frac{\partial \psi_{1}}{\partial y} \rightarrow 0 \quad \text { as } \quad y \rightarrow \infty(0 \leqslant x \leqslant \pi) .
\end{gathered}
$$

We can determine $\mu_{1}$ by applying a solvability condition to the system (3.8)-(3.11). Suppose that we can find a non-zero solution $\phi(x, y)$ of the equation

$$
\nabla^{2} \phi+2 \mathrm{e}^{-2 \psi_{0}} \phi=0
$$


with

$$
\frac{\partial \phi}{\partial n}=0 \quad \text { on } \partial R
$$

where $R$ is the semi-infinite rectangle $(0 \leqslant x \leqslant \pi, 0 \leqslant y \leqslant \infty)$ and where $\partial / \partial n$ is the normal derivative. Then

$$
\iint_{R}\left(\phi \nabla^{2} \psi_{1}-\psi_{1} \nabla^{2} \phi\right) \mathrm{d} x \mathrm{~d} y=\iint_{R} \phi\left[\nabla \rho_{1} \cdot \nabla \psi_{0}+2 \mathrm{e}^{-2 \psi_{0}}\left(\rho_{1}-\mu_{1} \psi_{0}\right)\right] \mathrm{d} x \mathrm{~d} y,
$$

and, since the left-hand side can be shown to vanish in view of (3.9)-(3.11) and (3.13), we have

$$
\iint_{R} \phi\left[\nabla \rho_{1} \cdot \nabla \psi_{0}+2 \mathrm{e}^{-2 \psi_{0}}\left(\rho_{1}-\mu_{1} \psi_{0}\right)\right] \mathrm{d} x \mathrm{~d} y=0 .
$$

We can verify that $\phi$ is given by

$$
\phi=\frac{\partial \psi_{0}}{\partial K},
$$

where $\psi_{0}$ is the Stuart vortex solution, expressed in terms of the original parameter $K$. (We are indebted to Professor T. Fokas for suggesting (3.16).) Substituting $\psi_{0}$ into (3.16) leads to

$$
\phi=\frac{\cosh y+K\left(K^{2}-1\right)^{-1 / 2} \cos x}{K \cosh y+\left(K^{2}-1\right)^{1 / 2} \cos x} .
$$

Clearly $\phi(x, y)=\phi(-x, y), \phi(\pi-x, y)=\phi(\pi+x, y)$ and $\phi(x, y)=\phi(x,-y)$, so that the boundary conditions (3.9) and (3.10) are satisfied. Next we note that as $y \rightarrow \infty$

$$
\phi=K^{-1}+O\left(\mathrm{e}^{-y}\right)
$$

so that the condition at infinity is satisfied. Finally, since the Stuart vortex solution $\psi_{0}(x, y, K)$ satisfies

$$
\nabla^{2} \psi_{0}=\exp \left(-2 \psi_{0}\right)
$$

it follows that

$$
\nabla^{2}\left(\frac{\partial \psi_{0}}{\partial K}\right)=-2 \frac{\partial \psi_{0}}{\partial K} \mathrm{e}^{-2 \psi_{0}} .
$$

To facilitate evaluation of the integrals in (3.15) we remark that it follows from (3.17) that

$$
\phi=\frac{(1-\epsilon)^{3 / 2}}{\epsilon}\left(\lambda+\frac{\rho_{1}}{\epsilon}\right), \quad \lambda=\frac{\epsilon}{1-\epsilon^{2}} .
$$

Then (3.15) can be written in the form

$$
I_{1}=\mu_{1} I_{2},
$$

where

$$
\begin{gathered}
I_{1}=\iint_{R}\left(\lambda+\frac{\rho_{1}}{\epsilon}\right)\left(\nabla \rho_{1} \cdot \nabla \psi_{0}+2 \mathrm{e}^{-2 \psi_{0}} \rho_{1}\right) \mathrm{d} x \mathrm{~d} y, \\
I_{2}=\iint_{R}\left(\lambda+\frac{\rho_{1}}{\epsilon}\right) 2 \mathrm{e}^{-2 \psi_{0}} \psi_{0} \mathrm{~d} x \mathrm{~d} y .
\end{gathered}
$$


These integrals can now be expressed in terms of weighted density integrals of the form

$$
J_{s}=\iint_{R} \rho_{1}^{s} \mathrm{e}^{-2 \psi_{0}} \mathrm{~d} x \mathrm{~d} y
$$

(see the Appendix) and

$$
\begin{gathered}
I_{1}=\lambda J_{1}+\frac{3}{2 \epsilon} J_{2}, \\
I_{2}=-\lambda J_{0}-\frac{1}{\epsilon} J_{1}+\frac{\pi \epsilon}{1-\epsilon^{2}} .
\end{gathered}
$$

The integrals $J_{s}$ can be evaluated using Mathematica and we find that $\mu_{1}=$ $\frac{1}{2}\left(1+\epsilon^{2}\right) /\left(1-\epsilon^{2}\right)$ so that

$$
\begin{aligned}
\mu & =1+\frac{M_{\infty}^{2}}{2}\left(\frac{1+\epsilon^{2}}{1-\epsilon^{2}}\right), \\
& =1+M_{\infty}^{2}\left(K^{2}-\frac{1}{2}\right),
\end{aligned}
$$

where the last equation follows from (3.5). Equation (3.28) confirms our interpretation of $\mu$ as an eigenvalue for the compressible Stuart vortex. This cannot be set equal to unity when $M_{\infty}>0$ but must be calculated as part of the overall solution. Comparison of (3.28) with numerical results will be given subsequently.

\section{Numerical solution}

Numerical solutions to (2.17) and (2.18) were sought using two different numerical methods, one spectral based and the other based on second-order finite differences. These are now described.

\subsection{Finite-difference solution}

We examine the formulation and reveal a difficulty by discretizing the boundaryvalue problem (2.17)-(2.18), (2.19)-(2.22). We introduce a mesh $((I-1) h,(J-1) h)$, $1 \leqslant I \leqslant M, 1 \leqslant J \leqslant N$, where $(M-1) h=\pi$ and take as unknowns $\psi_{d}(I, J)=$ $\psi((I-1) h,(J-1) h), \rho_{d}(I, J)=\rho((I-1) h,(J-1) h), d$ and $\mu$. We thus have a total of $2 M N+2$ unknowns. If we apply discretized versions of (2.17) and (2.18) at the mesh points (using (2.19)-(2.22) to obtain values of $\psi$ and $\rho$ at the ghost points adjacent to $\partial R$ ) we have $2 M N$ equations to satisfy. Equation (2.24) provides one extra equation, and so we are an equation short.

We provided an extra equation by insisting that the area integral of the vorticity over $R$ is equal to the line integral of the tangential velocity around $\partial R$. The tangential velocity is zero everywhere on $\partial R$ except at infinity, where it has unit value over a distance $\pi$. Thus we have

$$
\iint_{R} \omega(x, y) \mathrm{d} x \mathrm{~d} y=-\pi,
$$

where $\omega$ is obtained from

$$
\omega(x, y)=-\rho(x, y) \mathrm{e}^{-2 \mu \nu(x, y)} .
$$


Expressing (4.1)-(4.2) as an integral over the mesh leads to the condition that

$$
\sum_{I=1}^{M} \sum_{J=1}^{N} W(I, J) \rho_{d}(I, J) \mathrm{e}^{-2 \mu \psi_{d}(I, J)}=\pi,
$$

where $W(I, J)$ is a weighting function. We can thus close the system.

In practice three-point centred differences were used and the resulting system of nonlinear equations were solved by Newton's method. Unfortunately it was found that the Jacobian matrix was poorly conditioned. A possible explanation is that the boundary conditions (2.19)-(2.22) imply (4.1). Thus the Jacobian matrix will be singular in the limit $h \rightarrow 0$, and consequently will be ill-conditioned for $h$ small. Nevertheless, convergence was obtained, although it was not in all cases quadratic. Improved convergence was achieved by replacing the boundary condition (2.21) by its derivative with respect to $y$, thus eliminating the displacement thickness $d$. In the discrete version there are now only $2 M N+1$ unknowns and we can drop the vorticity integral condition (4.3). The convergence was quadratic except near terminal Mach numbers discussed in $\S 5$.

\subsection{Spectral method}

We considered it essential to solve the boundary-value problem by an independent method, and we now describe a spectral approach. This begins by writing $\psi$ and $\rho$ as

$$
\begin{gathered}
\psi=y \tanh y+d+\tilde{\psi}, \\
\rho=1+\tilde{\rho}, \\
\tilde{\psi}=\sum_{n=0}^{N-1} \sum_{m=0}^{M-1} a_{m n} \cos (m x) \phi_{2 n}(y), \\
\tilde{\rho}=\sum_{n=0}^{N-1} \sum_{m=0}^{M-1} b_{m n} \cos (m x) \phi_{2 n}(y),
\end{gathered}
$$

where $a_{m n}$ and $b_{m n}$ are coefficients to be determined,

$$
\phi_{k}(y)=\frac{1}{\left[2^{k} \Gamma(k+1) \sqrt{\pi}\right]^{1 / 2}} \exp \left(-\frac{y^{2}}{2}\right) H_{k}(y),
$$

and $H_{k}(y)$ is the Hermite polynomial of order $k$. The functions $\phi_{k}$ are orthonormal in $(-\infty, \infty)$ and the expansion

$$
g(y)=\sum_{n=0}^{\infty} d_{n} \phi_{n}(y), \quad d_{n}=\int_{-\infty}^{\infty} g(v) \phi_{n}(v) \mathrm{d} v,
$$

is convergent if the integral

$$
\int_{-\infty}^{\infty}|g(v)|^{2} \mathrm{~d} v
$$

exists. The expansions (4.6)-(4.7) satisfy the boundary conditions (2.19)-(2.20) provided $\tilde{\psi}$ and $\tilde{\rho}$ decay sufficiently rapidly as $y \rightarrow \infty$.

Equations (2.17) and (2.18) were satisfied by a collocation method. Equations (4.6) and (4.7) were substituted into each of (2.17) and (2.18). When these equations 


\begin{tabular}{rcc}
\hline$K$ & $d$ (numerical) & $d(\mathrm{exact})$ \\
1.1 & -0.597837 & -0.597837 \\
1.2 & -0.510823 & -0.510826 \\
1.4 & -0.356644 & -0.356675 \\
1.6 & -0.222979 & -0.223143 \\
1.8 & -0.104811 & -0.105360 \\
2.0 & 0.001391 & 0.0 \\
TABLE 1. Calculated values of $d,[M, N]=[40,30]$ compared exact results, $M_{\infty}=0$. \\
\hline
\end{tabular}

are satisfied at the collocation points $\left(x_{i}, y_{j}\right), i=1, \ldots M, j=1, \ldots N$, where $x_{i}=$ $\pi\left(i-\frac{1}{2}\right) / M$ are the zeros of $\cos (M x)$ and $y_{j}$ is the $j$ th zero of $H_{2 N}(y), 2 \times M \times N$ nonlinear equations are obtained for the $2 \times M \times N+2$ unknowns $a_{m, n}, b_{m, n}, m=$ $0, \ldots, M-1, n=0, \ldots, N-1, d$ and $\mu$. One additional equation is supplied when (4.6)(4.7) are substituted into the circulation equations (4.1)-(4.2) and a further equation is obtained by specifying $f$, the mass flow in the cat's eye in (2.23).

This gives $2 \times M \times N+2$ equations for an equivalent number of unknowns. These were solved by a standard Newton method with analytical evaluation of the Jacobian, which is full. The problem with ill-conditioning of the Jacobian found for the finitedifference method was also encountered in the spectral method. This did not prove fatal but did produce first-order rather than quadratic convergence. All numerical solutions reported below have average residual less than $10^{-12}$.

\section{Results and discussion}

In what follows, except where otherwise noted, all numerical results discussed were obtained with the spectral method. The continuation of the Stuart vortex from $M_{\infty}=0$ was obtained by first fixing $K$ and calculating $f$ from (2.23). A numerical solution was then obtained with $M_{\infty}=0$ using as an initial approximation a set of coefficients $a_{m n}$ calculated using (1.6), $b_{m n}=0, \mu=1$ and $d=\ln (K / 2)$. The compressible homentropic Stuart vortex array then admits a two-parameter family of solutions. Our strategy was to hold $f$ fixed, equivalent via (2.23) to holding $K$ fixed, and to continue numerically by incrementing $M_{\infty}$ in small intervals. Most spectral solutions reported below use $[M, N]=[30,40]$ although other values were used a check. Some $[60,60]$ spectral solutions, the highest resolution that could be achieved with available computing resources, were also obtained.

Accuracy was tested by comparing numerical results with the analytic solution at $M=0$, by comparing numerical solutions for $\mu$ with (3.29) at small $M_{\infty}$, and by comparing the results of the finite-difference and spectral calculations. Table 1 shows a comparison of the calculated values of $d$ (spectral method) with the exact $d=\ln (K / 2)$ at $M=0$. There is six-figure agreement at $K=1.1$ (found also for $\psi(x, y))$ but this declines to three-figure agreement at $K=2.0$. This is because as $K$ increases the vortex cores become more concentrated, with higher gradients - at $K=2$ the vorticity ratio $\left|\omega_{2} / \omega_{1}\right|=\left|\omega_{2}(0, \pi) / \omega_{1}(0,0)\right|$ is 194.28 .

Numerical solutions were obtained with $K=1.005,1.01,1.05,1.1,1.2,1.4,1.5$, 1.6, 1.8, 2.0 for a range of $M_{\infty}$ varying from $M_{\infty}=0$ to a maximum value which will be discussed subsequently. Figures 1-4 show contour plots of $\psi, \rho$, the local 


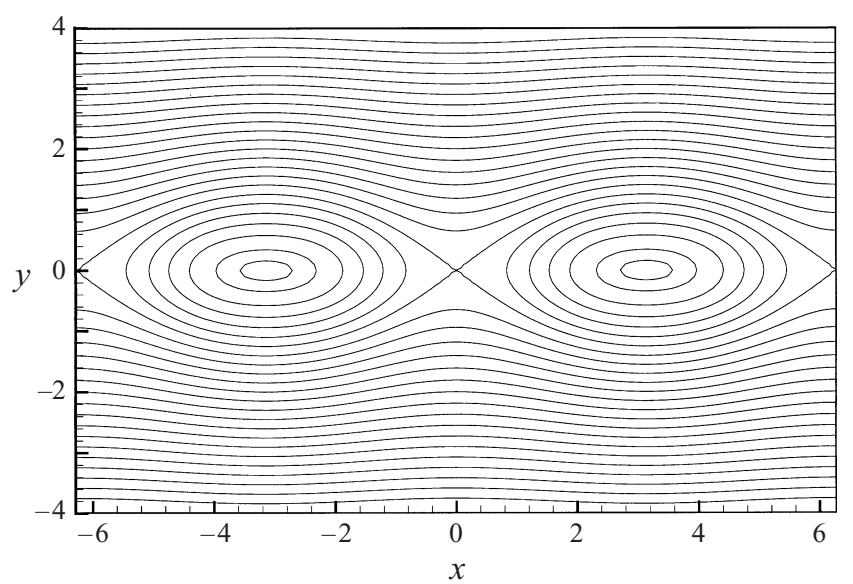

Figure 1. Compressible Stuart vortex $K=1.1, M_{\infty}=0.677$. Streamlines.

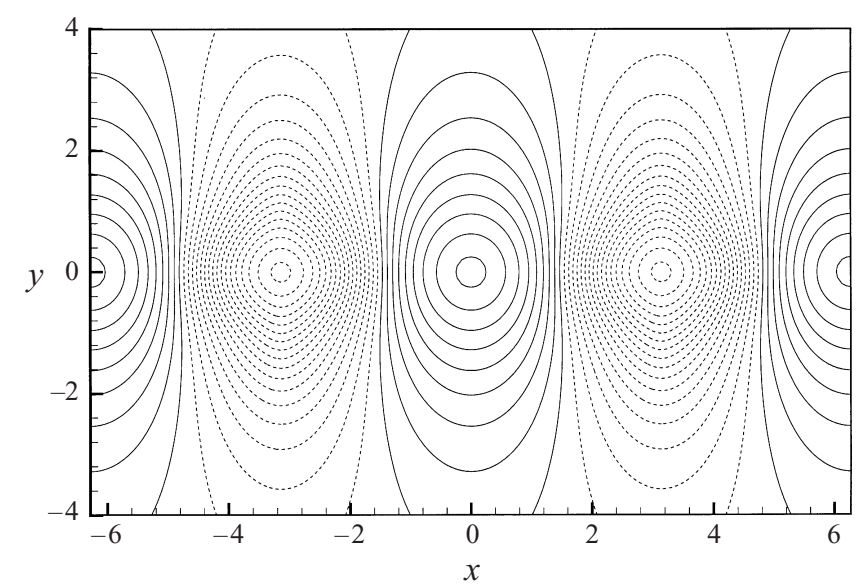

FIgURE 2. Compressible Stuart vortex $K=1.1, M_{\infty}=0.677$. Contours of density. Solid lines $\rho>\rho_{\infty}$. Dotted lines $\rho<\rho_{\infty}$.

Mach number

$$
M_{l}=M_{\infty} \frac{\left((\partial \psi / \partial x)^{2}+(\partial \psi / \partial y)^{2}\right)^{1 / 2}}{\rho^{(1+\gamma) / 2}},
$$

and $\nabla \cdot \boldsymbol{u}$ for $K=1.1, M=0.677$. The maximum Mach number $M_{\max }=\operatorname{Max}_{R}\left(M_{l}\right)$ occurs almost on the cat's eye boundary at $x=(2 n-1) \pi$ for $n=0, \pm 1, \pm \ldots$ Figures 5-10 summarize the effect of compressiblity on the Stuart vortex at fixed $K$. Plotted are $\mu$ (figure 5), $d$ (figure 6 ), the vorticity ratio $\left|\omega_{2} / \omega_{1}\right|$ (figure 7 ), the density ratio $\rho_{2} / \rho_{1}=\rho(\pi, 0) / \rho(0,0)$ (figure 8 ), the vorticity thickness

$$
\delta\left(K, M_{\infty}\right)=-\frac{1}{\pi} \iint_{R ; y>0} y \omega(x, y) \mathrm{d} x \mathrm{~d} y,
$$

(figure 9) and $M_{\max }$ (figure 10). The broad effects of increasing $M_{\infty}$ at fixed $K$ are to decrease $\rho_{2} / \rho_{1}$ and $\delta$ while increasing $M_{\max }$ and $\left|\omega_{2} / \omega_{1}\right|$. For $M_{\infty}=1.8,2.0$, the calculations show increases in $\left|\omega_{2} / \omega_{1}\right|$ by more than two orders of magnitude over a small range of $M_{\infty}$. This occurs because the vorticity eigenvalue $\mu$ increases faster 


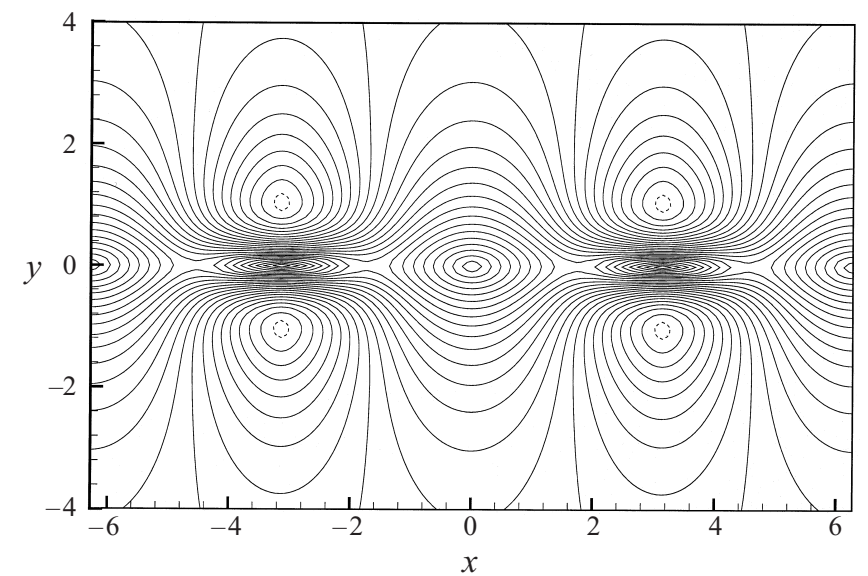

FIgURE 3. Compressible Stuart vortex $K=1.1, M_{v}=0.677$. Contours of constant Mach number. Solid lines $M_{l}<1$, dashed lines $M_{l}>1$.

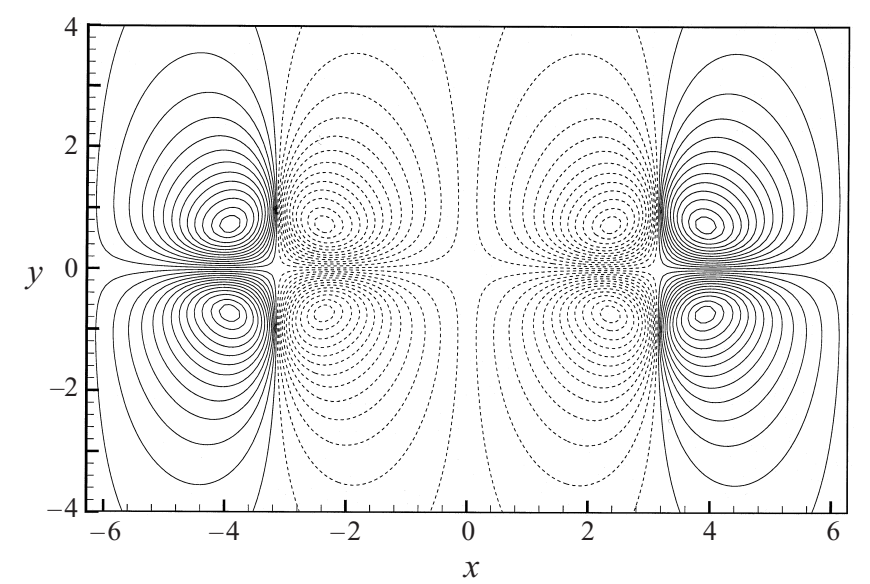

Figure 4. Compressible Stuart vortex $K=1.1, M_{v}=0.677$. Contours of $\nabla \cdot \boldsymbol{u}$. Solid lines $\nabla \cdot \boldsymbol{u}<0$. Dashed lines $\nabla \cdot \boldsymbol{u}>0$.

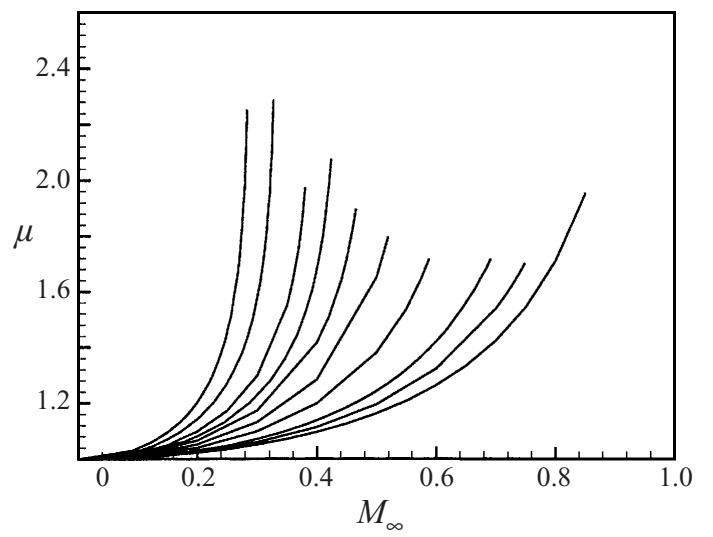

FIGURE 5. Vorticity constant $\mu$ versus $M_{\infty} . K=1.005,1.01,1.05,1.1,1.2,1.4,1.5,1.6,1.8,2.0$, $K$ increasing left to right. 


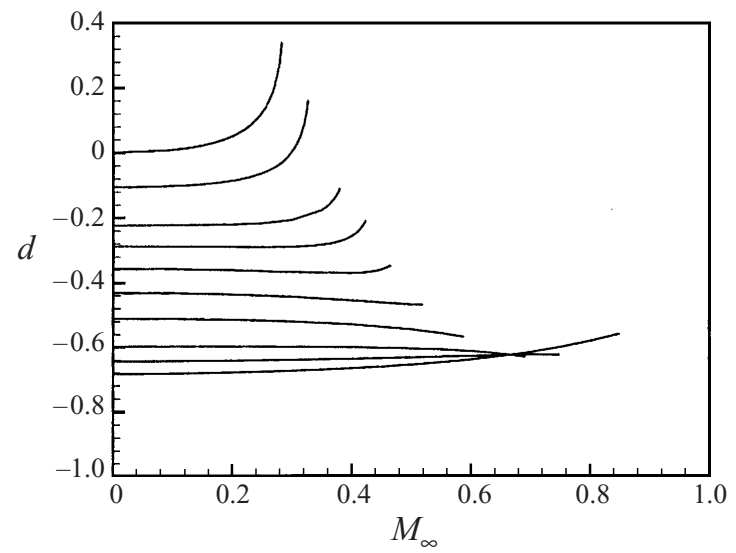

FIGURE 6. Displacement thickness $d$ versus $M_{\infty} . K$ increasing bottom to top. Values of $K$ as in figure 5 .

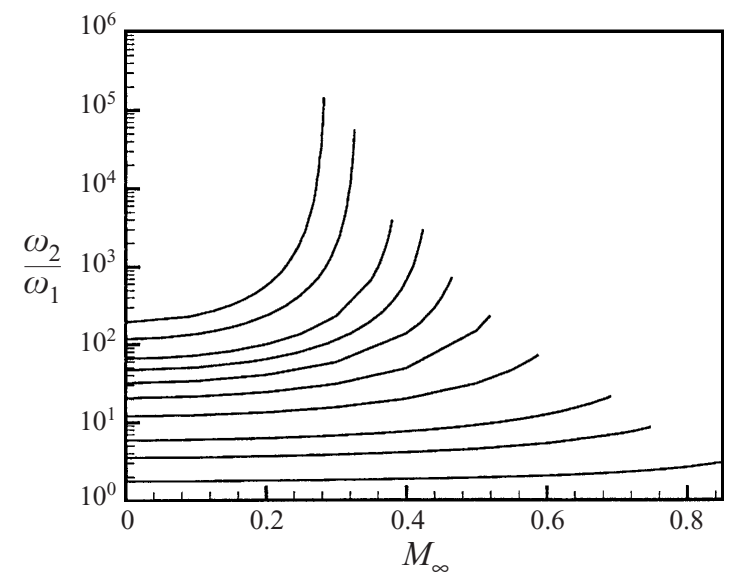

FIGURE 7. Vorticity ratio $\omega_{2} / \omega_{1} . K$ increasing bottom to top. Values of $K$ as in figure 5.

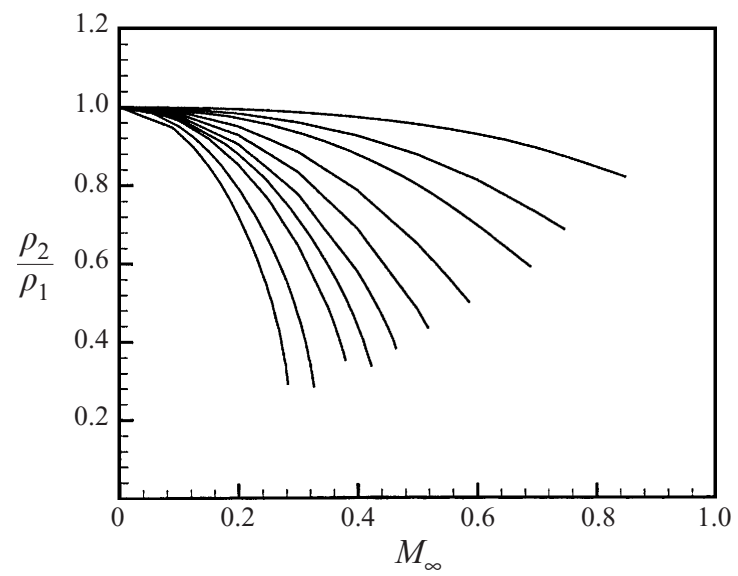

Figure 8. Density ratio $\rho_{2} / \rho_{1}$. $K$ decreasing left to right. Values of $K$ as in figure 5 . 


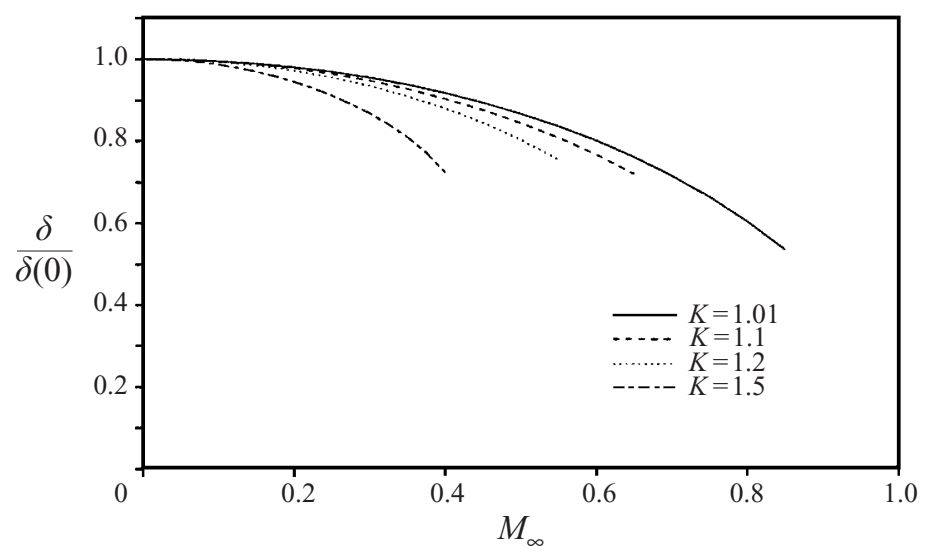

FIGURE 9. Vorticity thickness $\delta\left(M_{\infty}\right) / \delta(0)$.

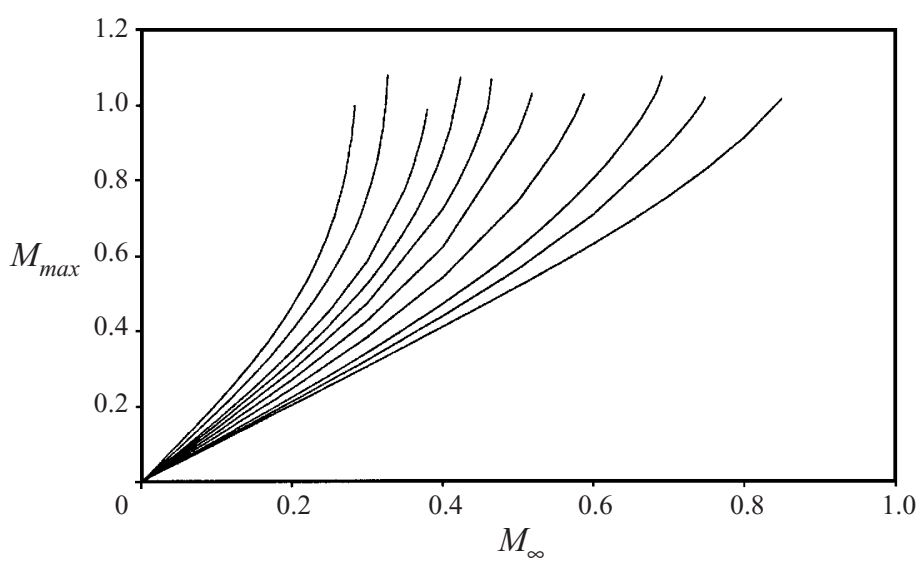

FIgURE 10. Maximum Mach number. $K$ decreasing left to right. Values of $K$ as in figure 5 .

(a) $\begin{array}{lcl}M_{\infty} & \mu \text { (numerical) } & \mu(\mathrm{R}-\mathrm{J}) \\ 0 & 1.000000 & 1.0 \\ 0.1 & 1.007175 & 1.0071 \\ 0.2 & 1.029649 & 1.0284 \\ 0.3 & 1.070616 & 1.0639 \\ 0.4 & 1.136492 & 1.1136\end{array}$

\begin{tabular}{|c|c|c|c|}
\hline (b) & $M_{\infty}$ & $\mu$ (numerical) & $\mu(\mathrm{R}-\mathrm{J})$ \\
\hline & 0 & 1.000000 & 1.0 \\
\hline & 0.1 & 1.014977 & 1.0146 \\
\hline & 0.2 & 1.065001 & 1.0584 \\
\hline & 0.3 & 1.171643 & 1.1314 \\
\hline & 0.4 & 1.416803 & 1.2336 \\
\hline
\end{tabular}

TABLE 2. Calculated values of $\mu,[M, N]=[40,30]$ compared with results from Rayleigh-Janzen expansion. (a) $K=1.1$. (b) $K=1.4$

than the density at the vortex centre decreases. It is of interest to note that the ratio $\delta\left(K, M_{\infty}\right) / \delta(K, 0)$ decreases with increasing $M_{\infty}$ for all $K$ considered.

In table 2 we show the computed values of $\mu$ for $K=1.1$ compared to the predictions of the Rayleigh-Janzen expansion, equation (3.29). This is satisfactory at small $M_{\infty}$, giving us confidence in both our numerics and the Rayleigh-Janzen analysis, when $M_{\infty}>0$, but there are discrepancies at larger $M_{\infty}$. We remark that the expansion (3.1) and (3.2), where $\psi_{1}$ is determined by the boundary-value problem 


$\begin{array}{lllccc}K & M_{\infty} & \delta \rho(\operatorname{mean}) & \delta \rho(\max ) & \delta \psi(\operatorname{mean}) & \delta \psi(\max ) \\ 1.1 & 0.2 & 1.2 \times 10^{-7} & 8.0 \times 10^{-7} & 6.9 \times 10^{-7} & 3.6 \times 10^{-6} \\ 1.1 & 0.4 & 8.4 \times 10^{-7} & 3.8 \times 10^{-6} & 5.4 \times 10^{-7} & 1.6 \times 10^{-6} \\ 1.1 & 0.68 & 4.5 \times 10^{-5} & 1.3 \times 10^{-3} & 1.4 \times 10^{-5} & 3.0 \times 10^{-5}\end{array}$

TABLE 3. Comparison of solutions for $\psi(x, y)$ and $\rho(x, y)$ obtained by the spectral method with $[M, N]=[40,30]$ and the finite-difference method using Richardson extrapolation from $21 \times 81$ and $41 \times 61$ grids. The mean - taken over the domain $(0<x, \pi, 0<y<\pi)$-and the maximum of the absolute value of the difference is displayed.

(3.8)-(3.11), will not satisfy the flux condition (2.23). This means that the RayleighJanzen expansion is yielding a slightly different compressible continuation of the Stuart vortex. The only parameter at our disposal is $K$ and, if we wish to reproduce the solution found numerically, we must choose $K$ so that (2.23) is satisfied. Now the flux condition is violated because $\psi_{1}(0,0)-\psi_{1}(\pi, 0)$ is non-zero (in general) and to compensate for this we must perturb $\psi_{0}$ by $O\left(M_{\infty}^{2}\right)$, which requires a shift in $K$ of the same order. The fields $\psi_{1}$ and $\rho_{1}$ will be perturbed by $O\left(M_{\infty}^{2}\right)$ and (3.15) shows that the change in $\mu_{1}$ is $O\left(M_{\infty}^{2}\right)$ also. Thus the effect on $\mu$ is $O\left(M_{\infty}^{4}\right)$. It is thus legitimate to compare the numerical and analytical predictions of $\mu$, provided $O\left(M_{\infty}^{4}\right)$ is neglected. In table 3 we show a comparison of solutions obtained using the spectral and finite difference methods for $K=1.1$ and for three values of $M_{\infty}$. This is made on the basis of the mean and the maximum of the absolute difference between computed solutions over the domain indicated in that table. This comparison is satisfactory and further supports our confidence in our numerical and analytical results.

It was found that all branches followed by continuation in $M_{\infty}$ with $K$ fixed terminated at a point where $M_{\max }$ was somewhat larger than unity. This can be seen in figure 10. That this was not a result of the choice of $M_{\infty}$ as continuation parameter was verified by employing arclength continuation in the space of all the unknowns. With fixed arclength increment, and using fourth-order extrapolation in arclength to estimate the next approximation, this led to exceedingly small increments in all parameters but no change in the values of $M_{\infty}$ at which the Newton method failed to find solutions. From this we conclude that there is not a turning point along say a branch continued in $M_{\infty}$, and that the branch termination was genuine.

One aim of the present investigation was to search for numerical solutions to (2.17)(2.18) corresponding to smooth transonic flow. It is well known (Morawetz 1956, 1957, 1958) that solutions of the two-dimensional Euler equations corresponding to smooth irrotational, transonic flow past fixed airfoil shapes are, if they exist, isolated. Moore \& Pullin (1987) and Ardalan et al. (1995) found families of smooth transonic flow for compressible flows with constant-pressure vortex cores. They interpreted this non-conformity with Morawetz's results as due to the free-boundary character of the hollow-core compressible vortex, the boundary of which was able to adjust its shape when $M_{\infty}$ changed. Moore \& Pullin (1998), however, find no solutions with smooth transonic flow for the compressible Hill's spherical vortex.

To investigate this question for the compressible Stuart vortex, some high-resolution solutions were obtained with $K=1.1,[M, N]=[60,60]$ at values of $M_{\infty}$ near the onset of locally supersonic flow. Table 4 shows $M_{\max }$ against $M_{\infty}$ for three different resolutions, $[M, N]=[20,60],[40,60]$ and $[60,60]$. All three sets and the finitedifference calculations show the onset of locally supersonic flow for $M_{\infty}$ in the range 


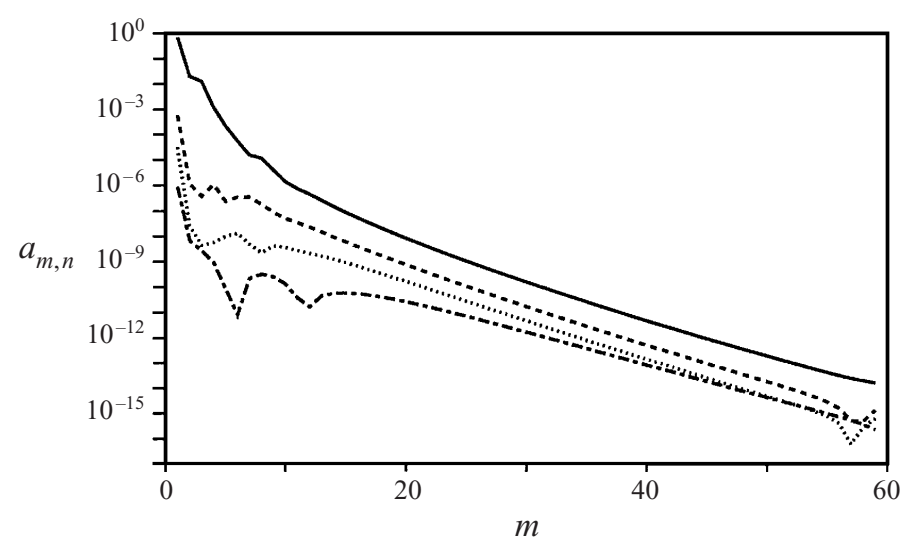

Figure 11. $a_{m, n}$ vs. $m ; M_{\infty}=0.667, n=25$ (solid), 35 (dashed), 45 (dotted), 55 (dash-dot).

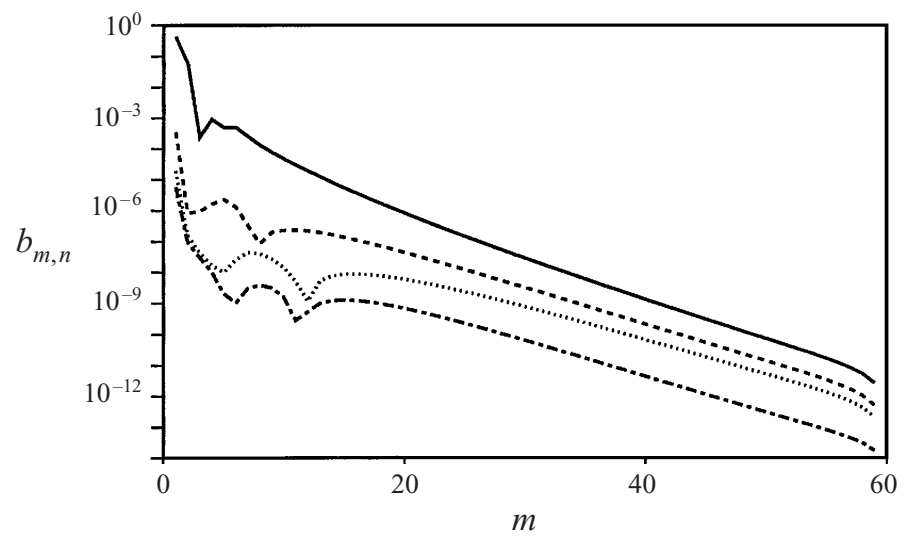

FiguRE 12. $b_{m, n}$ vs. $m ; M_{\infty}=0.667, n=25,35,45,55$. For key see figure 11.

$0.674<M_{\infty}<0.675$. A run with a $71 \times 281$ grid at $M_{\infty}=0.68$ failed to converge. Three-point interpolation gives $M_{\max }=1.0000$ at $M_{\infty} \approx 0.6746$.

In figures 11 and 12 we plot the decay of the coefficients $\left|a_{m n}\right|$ and $\left|b_{m n}\right|$ of (4.6)(4.7) with $m$, for several values of $n$, with $M_{\infty}=0.667$, at which value the flow is everywhere subsonic with $M_{\max }=0.9745$. There appears to be exponential decay of the coefficients indicating convergence of the series in (4.6)-(4.7). In order to determine where exponential convergence is lost, a form for the variation of the magnitude of the coefficients

$$
\left|a_{m n}\right| \sim \exp \left[-\alpha\left(n, M_{\infty}\right) m\right], \quad\left|b_{m n}\right| \sim \exp \left[-\beta\left(n, M_{\infty}\right) m\right],
$$

was assumed, and least-squares fits of $\log \left(\left|a_{m n}\right|\right), \log \left(\left|b_{m n}\right|\right)$ versus $m, 25 \leqslant m \leqslant 55$ (to avoid end effects) were made for several values of $n$. The slopes of these fits give estimates of $\alpha\left(n, M_{\infty}\right), \beta\left(n, M_{\infty}\right)$, which are plotted in figures 13 and 14 respectively versus $M_{\infty}$ for several $n$. Both $\alpha$ and $\beta$ seem to pass through zero near $M_{\infty} \approx 0.678$, indicating loss of exponential convergence for $M_{\infty}>0.678$. From table $4, M_{\max }=$ 1.014 at this $M_{\infty}$. This provides some evidence for the existence of a very small band, $0.6746<M_{\infty}<0.678$, in which locally supersonic flow exists. Figures 15 and 16 show the decay of $\left|a_{m n}\right|$ and $\left|b_{m n}\right|$ respectively with $m$, for $M_{\infty}=0.677$, at which $M_{\max }=1.009$. The variation of the magnitude of the coefficients with $m$ shows a 


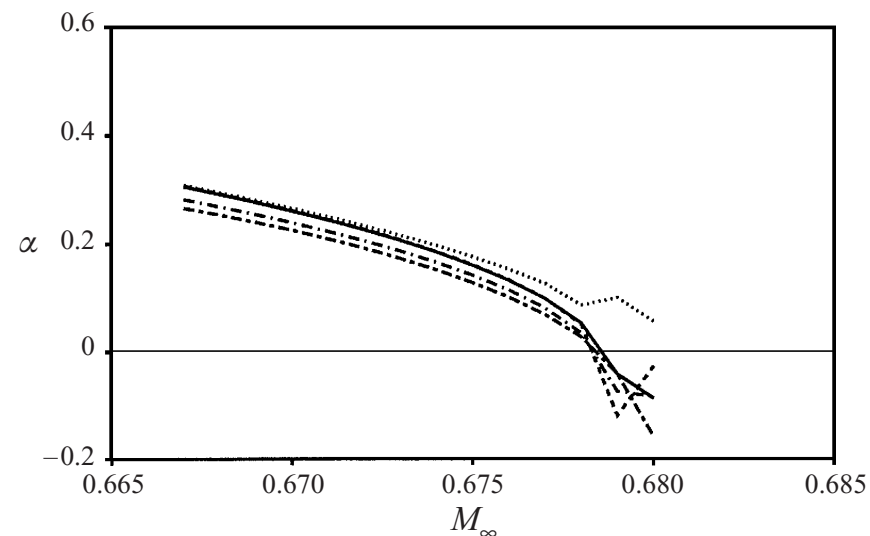

Figure 13. $\alpha$ vs. $M_{\infty} ; n=30$ (solid), 40 (dashed), 50 (dotted), 59 (dash-dot).

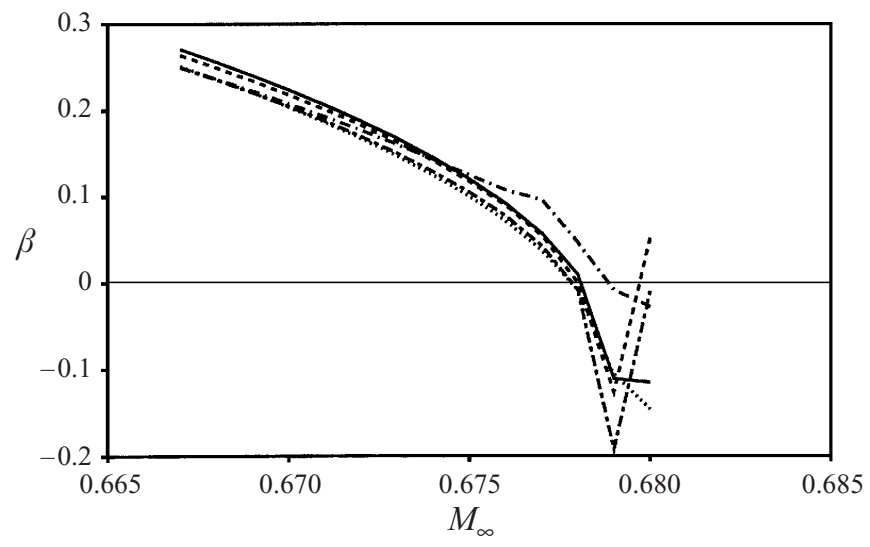

Figure $14 . \beta$ vs. $M_{\infty} ; n=30,40,50,59$. For key see figure 13 .

$\begin{array}{lrccc}M_{\infty} & {[20,60]} & {[40,60]} & {[60,60]} & \mathrm{RE} \\ 0.667 & 0.974521 & 0.974534 & 0.974534 & 0.9745147 \\ 0.668 & 0.977709 & 0.977726 & 0.977726 & 0.9777113 \\ 0.669 & 0.980934 & 0.980956 & 0.980957 & 0.9809420 \\ 0.670 & 0.984200 & 0.984230 & 0.984230 & 0.9842140 \\ 0.671 & 0.987509 & 0.987550 & 0.987550 & 0.9875333 \\ 0.672 & 0.990866 & 0.990922 & 0.990923 & 0.9909050 \\ 0.673 & 0.994275 & 0.994353 & 0.994354 & 0.9943357 \\ 0.674 & 0.997741 & 0.997852 & 0.997856 & 0.9978353 \\ 0.675 & 1.001271 & 1.001434 & 1.001444 & 1.0014180 \\ 0.676 & 1.004874 & 1.005120 & 1.005147 & 1.0051123 \\ 0.677 & 1.008560 & 1.008948 & 1.009042 & 1.0089787 \\ 0.678 & 1.012342 & 1.012995 & 1.013536 & 1.0134110 \\ 0.679 & 1.016238 & 1.017460 & 1.015035 & 1.0165597 \\ 0.680 & 1.020273 & 1.023075 & 1.020522 & 1.0225863\end{array}$

TABLE 4. Calculated maximum local Mach number $M_{\max }$, spectral method with various resolutions $[M, N]$ and Richardson extrapolation $(\mathrm{RE})$ from $31 \times 121$ and $61 \times 241$ grids for the finite-difference calculation. 


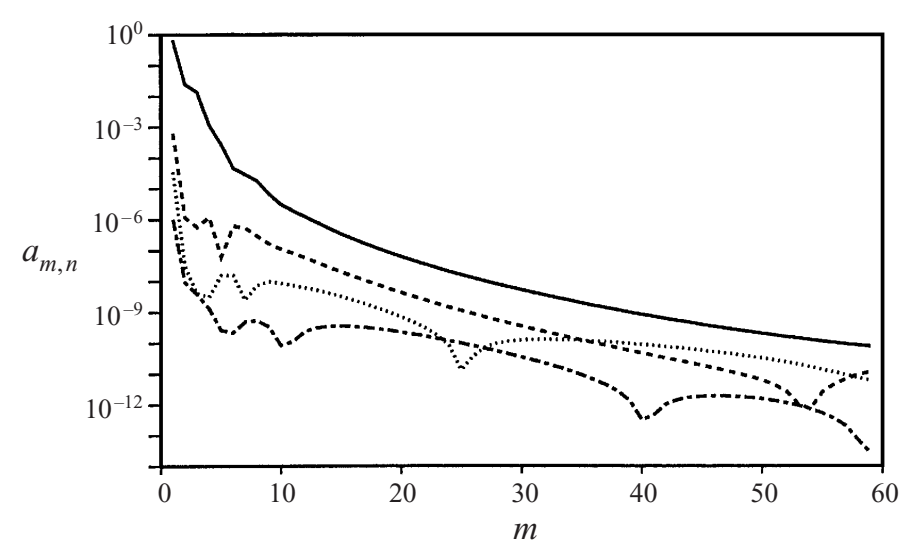

FIGURE 15. $a_{m, n}$ vs. $m ; M_{\infty}=0.677, n=25$ (solid), 35 (dashed), 45 (dotted), 55 (dash-dot).

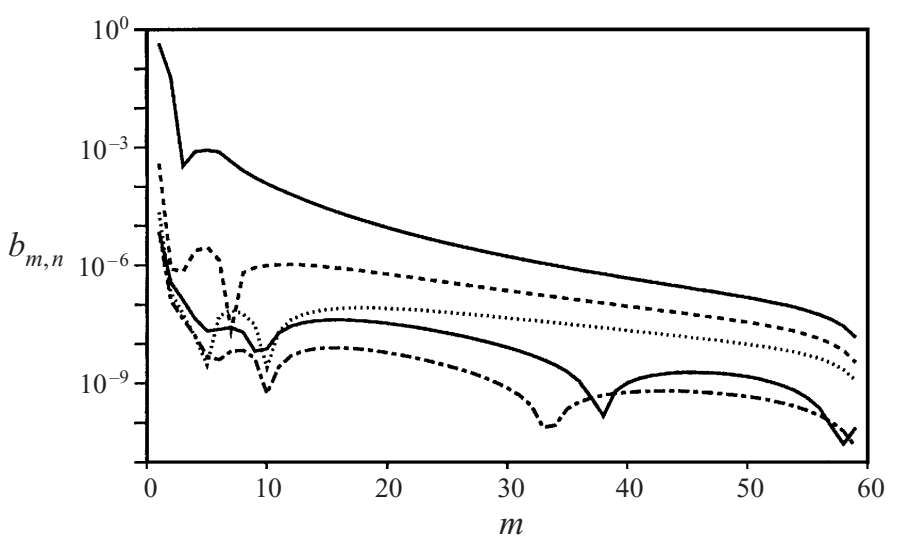

FIGURE 16. $b_{m, n}$ Vs. $m ; M_{\infty}=0.677, n=25,35,45,55$. For key see figure 15 .

lack of smoothness compared with figures 11 and 12 but there is apparent slow exponential decay, albeit with some small upward curvature for the $\left|a_{m n}\right|$.

In figures 17 and 18 we show contours of $1-M_{l}$ for both $M_{\infty}=0.677$ and $M_{\infty}=0.680$, windowed on the region near $(x, y)=(\pi, 1.04)$, near where locally supersonic flow is indicated. For $M_{\infty}=0.677$ the transonic flow region is of surprisingly large extent (given the small value of $M_{\max }-1$ ), and both this figure and similar plots of $\rho$ and $\nabla \cdot \boldsymbol{u}$ (not shown) indicate smooth flow. In contrast, the $1-M_{l}$ contours for $M_{\infty}=0.680$, at which exponential convergence of the cosine series seems to be lost, show irregularity. Note the separation into two local minima of $1-M_{l}$ for this case. This may indicate the formation of incipient weak shocks with shock strength $M_{l}^{2}-1=O\left(10^{-2}\right)$. This would explain the convergence failure for the cosine series. We recall that owing to enforced symmetry about $x=\pi$, such weak shocks must also be symmetrical, corresponding to a compression/expansion pair.

\section{Concluding remarks}

We have obtained analytical and accurate spectral and finite-difference numerical solutions corresponding to the continuation to finite Mach number of a family 


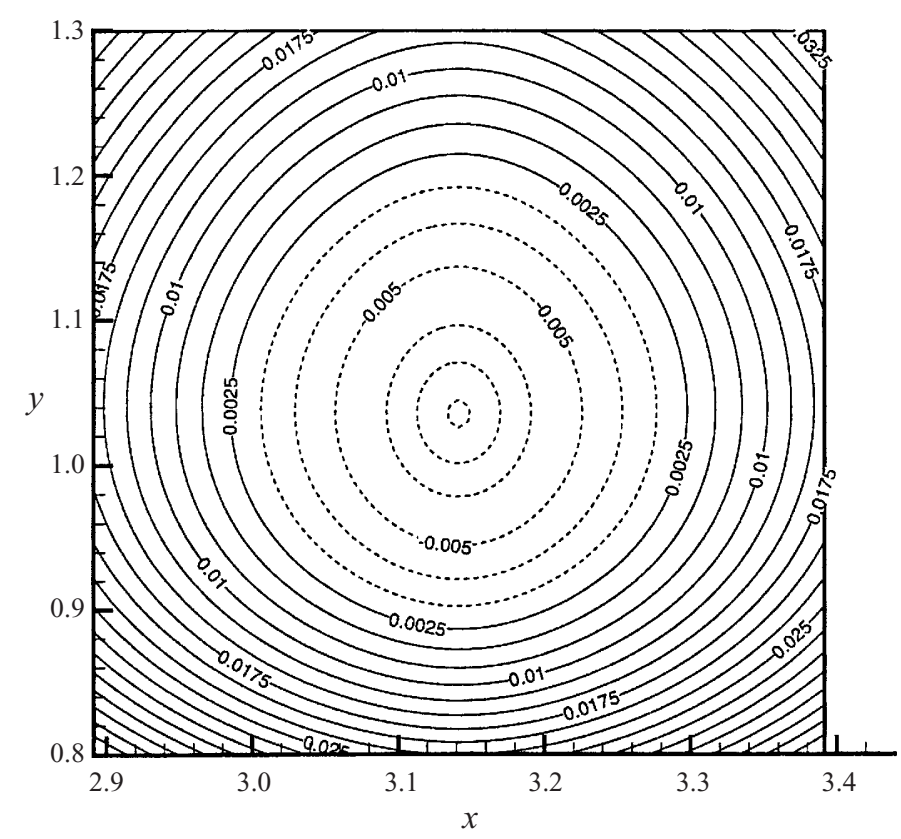

Figure 17. Contours of local Mach number $1-M_{l} . M_{\infty}=0.677$. Solid lines, $1-M_{l}>0$. Dashed lines, $1-M_{l}<0$.

of Stuart vortex solutions of the inviscid Euler equations in two dimensions. Unexpectedly, the homentropic continuation of the incompressible Stuart vortex into the compressible regime required the introduction of an effective eigenvalue into the vorticity-density-streamfunction relationship, whose value for fixed Mach number (at infinity) and mass-flow rate within the recirculating bubble region or vortex 'cat's eye' must be determined as part of the overall solution. The numerical solutions indicated that when the Mach number at infinity was increased at fixed mass-flow rate, the ratio of the vorticity at the vortex centre to that at the stagnation point separating the cat's eye regions, increased very rapidly, while the corresponding density ratio decreased.

It was found that solution branches with fixed mass-flow rate always terminated when the maximum local Mach number just exceeded unity. For one value of the mass-flow rate corresponding to a value of the Stuart vortex parameter $K=1.1$, we found some evidence for the existence of a very small range of free-stream Mach numbers $0.6746<M_{\infty}<0.678$ corresponding to smooth transonic flow. This non-conformity with the results of Morawetz $(1956,1957,1958)$ may be due to the rotational character of the present Stuart vortex flow. For $M_{\infty}>0.678$ we speculate that weak, almost-entropy-preserving shocks may appear.

We remark that (2.17) and (2.18) with the boundary conditions (2.19)-(2.22) do not define a unique continuation of the Stuart vortex solution to finite Mach number. Moore \& Pullin (1998) discuss several admissible continuations of the incompressible Hill's spherical vortex flow to give solutions of the steady compressible Euler equations. These include the assumption of homethalpic flow in place of the present homentropic flow and the application of a transformation which generates new solutions of the steady Euler equations from a given solution (Munk \& Prim 1947). These methods can be applied to the present Stuart vortex flow. Finally, we have 


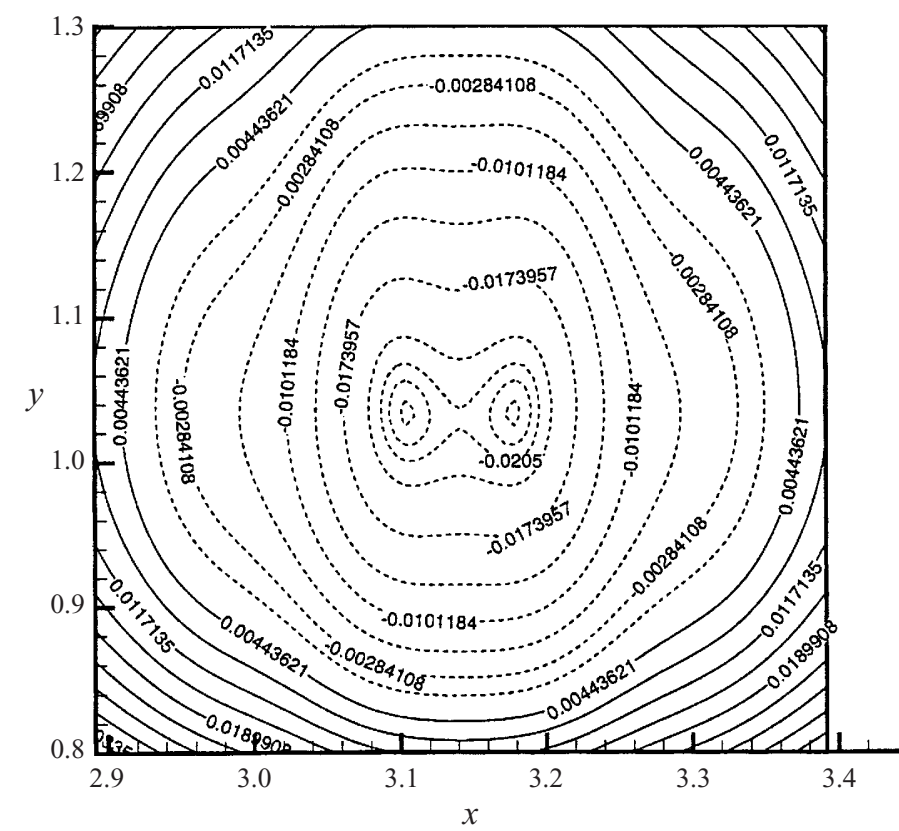

FIGURE 18. Contours of local Mach number $1-M_{l} . M_{\infty}=0.680$. Solid lines, $1-M_{l}>0$. Dashed lines, $1-M_{l}<0$.

neither considered the existence of solution branches not attached to the incompressible limit, such as a bifurcation from infinity, nor investigated a formulation which would allow for possible bifurcation to solutions not symmetrical about $x= \pm n \pi, n$ integer.

\section{Appendix}

We start by considering the integrand of $I_{1}$. First we remark that

$$
\nabla \rho_{1} \cdot \nabla \psi_{0}=\nabla \cdot\left(\rho_{1} \nabla \psi_{0}\right)-\rho_{1} \nabla^{2} \psi_{0}
$$

so that in view of (3.19)

$$
\nabla \rho_{1} \cdot \nabla \psi_{0}=\nabla \cdot\left(\rho_{1} \nabla \psi_{0}\right)-\rho_{1} \mathrm{e}^{-2 \psi_{0}} .
$$

Hence

$$
I_{1}=\iint_{R}\left(\lambda+\frac{\rho_{1}}{\epsilon}\right)\left(\nabla \cdot\left(\rho_{1} \nabla \psi_{0}\right)+\rho_{1} \mathrm{e}^{-2 \psi_{0}}\right) \mathrm{d} x \mathrm{~d} y .
$$

Now, denoting the unit outward normal to $\partial R$ by $\boldsymbol{n}$,

$$
\iint_{R} \nabla \cdot\left(\rho_{1} \nabla \psi_{0}\right) \mathrm{d} x \mathrm{~d} y=\int_{\partial R} \rho_{1}\left(\boldsymbol{n} \cdot \nabla \psi_{0}\right) \mathrm{d} \sigma,
$$

where $\sigma$ is arclength on $\partial R$. The integral on the right of (A 4) is zero since $\partial \psi_{0} / \partial n=0$ on the base and on the vertical sides of $R$ and $\rho_{1}$ is exponentially small and $\partial \psi_{0} / \partial n$ is bounded as $y \rightarrow \infty$. Hence

$$
I_{1}=\iint_{R}\left(\lambda+\frac{\rho_{1}}{\epsilon}\right) \rho_{1} \mathrm{e}^{-2 \psi_{0}} \mathrm{~d} x \mathrm{~d} y+\frac{1}{\epsilon} \iint_{R} \rho_{1} \nabla \cdot\left(\rho_{1} \nabla \psi_{0}\right) \mathrm{d} x \mathrm{~d} y .
$$


We can show that

$$
\rho_{1} \nabla \cdot\left(\rho_{1} \nabla \psi_{0}\right)=\nabla \cdot\left(\frac{1}{2} \rho_{1}^{2} \nabla \psi_{0}\right)+\frac{1}{2} \rho_{1}^{2} \nabla^{2} \psi_{0} .
$$

If we substitute (A 6) into the integral on the right of (A 5) we find that

$$
I_{1}=\iint_{R}\left(\lambda+\frac{\rho_{1}}{\epsilon}\right) \rho_{1} \mathrm{e}^{-2 \psi_{0}} \mathrm{~d} x \mathrm{~d} y+\frac{1}{2 \epsilon} \iint_{R} \rho_{1}^{2} \mathrm{e}^{-2 \psi_{0}} \mathrm{~d} x \mathrm{~d} y,
$$

where we have again used (3.10); the integral of the first term on the right of (A 6) can be seen to be zero since

$$
\iint_{R} \nabla \cdot\left(\frac{1}{2} \rho_{1}^{2} \nabla \psi_{0}\right) \mathrm{d} x \mathrm{~d} y=\int_{\partial R} \frac{1}{2} \rho_{1}^{2}\left(\boldsymbol{n} \cdot \nabla \psi_{0}\right) \mathrm{d} \sigma,
$$

and the boundary integral is zero. Introducing the definition of $J_{s}$ given in (3.25) enables us to express $I_{1}$ in the form

$$
I_{1}=\lambda J_{1}+\frac{3}{2 \epsilon} J_{2}
$$

To reduce $I_{2}$ we introduce the function

$$
\hat{\phi}=\lambda+\frac{\rho_{1}}{\epsilon}=\frac{\epsilon \phi}{\left(1-\epsilon^{2}\right)^{3 / 2}},
$$

so that

$$
I_{2}=2 \iint_{R} \hat{\phi} \psi_{0} \mathrm{e}^{-2 \psi_{0}} \mathrm{~d} x \mathrm{~d} y
$$

Now, since $\hat{\phi}$ is a constant multiple of $\phi$,

$$
\nabla^{2} \hat{\phi}=-2 \mathrm{e}^{-2 \psi_{0}} \hat{\phi}
$$

and so

$$
I_{2}=-\iint_{R} \nabla^{2} \hat{\phi} \psi_{0} \mathrm{~d} x \mathrm{~d} y .
$$

But for any pair of functions, for which the integrals converge, Green's identity states that

$$
\iint_{R}\left(\psi_{0} \nabla^{2} \hat{\phi}-\hat{\phi} \nabla^{2} \psi_{0}\right) \mathrm{d} x \mathrm{~d} y=\int_{\partial R} \psi_{0}(\boldsymbol{n} \cdot \nabla \hat{\phi}) \mathrm{d} \sigma-\int_{\partial R} \hat{\phi}\left(\boldsymbol{n} \cdot \nabla \psi_{0}\right) \mathrm{d} \sigma .
$$

The first boundary integral is zero. However

$$
\hat{\phi}=\rightarrow \lambda \quad \text { as } y \rightarrow \infty \quad(0 \leqslant x \leqslant \pi),
$$

and

$$
\frac{\partial \psi_{0}}{\partial y}=\rightarrow 1 \quad \text { as } y \rightarrow \infty \quad(0 \leqslant x \leqslant \pi),
$$

so that the contribution to the line integral from the upper boundary of $R$ is $\pi \lambda$. Thus on rearranging (A 14) and using (3.10)

$$
I_{2}=-\iint_{R} \hat{\phi} \mathrm{e}^{-2 \psi_{0}} \mathrm{~d} x \mathrm{~d} y+\pi \lambda,
$$

or

$$
I_{2}=-\lambda J_{0}-\frac{1}{\epsilon} J_{1}+\frac{\pi \epsilon}{1-\epsilon^{2}} .
$$


Finally, we substitute for $\psi_{0}$ from (3.4) and for $\rho_{1}$ from (3.7) to find

$J_{s}=\left(1-\epsilon^{2}\right) \epsilon^{s} \int_{0}^{\infty} \frac{4}{1+t^{2}}\left(\frac{1-t^{2}}{1+t^{2}}\right)^{s} \int_{0}^{1}\left(\frac{1+u^{2}}{1-u^{2}}+\epsilon \frac{1-t^{2}}{1+t^{2}}\right)^{-s-2} \frac{1}{1-u^{2}} \mathrm{~d} u \mathrm{~d} t, \quad$ (A 19) where $t=\tan \left(\frac{1}{2} x\right)$ and $u=\tan \left(\frac{1}{2} y\right)$. Mathematica gives

$$
\left.J_{0}=\pi, \quad J_{1}=-\frac{\pi \epsilon^{2}}{1-\epsilon^{2}}, \quad J_{2}=\frac{\pi \epsilon^{2}\left(1+3 \epsilon^{2}\right)}{3\left(1-\epsilon^{2}\right)^{2}} . \quad \text { (A 20, A } 21, \mathrm{~A} 22\right)
$$

\section{REFERENCES}

Ames, W. F. 1965 Nonlinear Partial Differential equations in Engineering. Academic.

Ardalan, K., Meiron, D. I. \& Pullin, D. I. 1995 Steady compressible vortex flows: the hollow-core vortex array. J. Fluid Mech. 301, 1-17.

Forsyth, A. R. 1959 Theory of Differential Equations, Vols. 5 \& 6. Dover.

Moore, D. W. \& Pullin, D. I. 1987 The compressible vortex pair. J. Fluid Mech. 185, 171-204.

Moore, D. W. \& Pullin, D. I. 1998 On steady compressible flows with compact vorticity; the compressible Hill's spherical vortex. J. Fluid Mech. 374, 285-303.

MorawetZ, C. S. 1956 On the non-existence of continuous transonic flows past airfoils. I. Commun. Pure Appl. Maths 9, 45-68.

Morawetz, C. S. 1957 On the non-existence of continuous transonic flows past airfoils. II. Commun. Pure Appl. Maths 10, 107-131.

Morawetz, C. S. 1958 On the non-existence of continuous transonic flows past airfoils. III. Commun. Pure Appl. Maths 11, 129-144.

MunK, M. \& PrIM, R. 1947 On the multiplicity of steady gas flows having the same streamline pattern. Proc. Natl Acad. Sci. 33, 137-141.

Stuart, J. T. 1967 On finite amplitude oscillations in mixing layers. J. Fluid Mech. 29, 417-440.

VAZSOnYI, A. 1945 On rotational gas flows. Q. J. Appl. Maths 3, 29-38. 